\title{
ADDITIVE POLYNOMIALS OVER PERFECT FIELDS
}

\author{
SALIH DURHAN
}

\section{INTRODUCTION}

A polynomial $f\left(x_{1}, \ldots, x_{n}\right)$ over a field $K$ is additive if

$$
f\left(a_{1}+b_{1}, \ldots, a_{n}+b_{n}\right)=f\left(a_{1}, \ldots, a_{n}\right)+f\left(b_{1}, \ldots, b_{n}\right)
$$

for all $a_{1}, \ldots, a_{n}, b_{1}, \ldots, b_{n} \in L$ where $L$ is any extension of $K$. In characteristic zero the only additive polynomials are the linear polynomials with constant term zero. In positive characteristic, $p$, additive polynomials are of the form

$$
\sum_{i=1}^{n} \sum_{j=0}^{k_{i}} a_{i j} x_{i}^{p^{j}}
$$

where $a_{i j} \in K$. Additive polynomials over valued fields in positive characteristic play an important role in understanding many algebraic and model theoretic properties of maximal fields of positive characteristic, see [7] for a thorough examination of the issue.

A subset $\mathcal{S}$ of a valued field $(K, v)$ has the optimal approximation property if for all $a \in K$, the set $\{v(s-a): s \in \mathcal{S}\}$ has a maximal element. By the image of a polynomial $f\left(x_{1}, \ldots, x_{n}\right)$ over $K$ we mean the set

$$
\left\{f\left(a_{1}, \ldots, a_{n}\right): a_{1}, \ldots, a_{n} \in K\right\} .
$$

As pointed out in [7], one natural question is: Does the image of every additive polynomial over a maximal valued field of positive characteristic have the optimal approximation property? It is shown in [8] that the image of an additive polynomial over $\mathbb{F}_{q}((t))$ (considered as a valued field with the $t$-adic valuation) has the optimal approximation property. Furthermore this elementary property is independent of the previously observed elementary properties of $\mathbb{F}_{q}((t))$, see [5]. In particular henselianity, algebraic maximality and even being defectless, in addition to algebraic maximality, does not imply the optimal approximation property for images of additive polynomials. However, when additive polynomials in one variable are considered algebraic maximality is sufficient, [6].

Theorem 1.1. A henselian valued field of characteristic $p>0$ is algebraically maximal if and only if the image of every additive polynomial in one variable has the optimal approximation property.

An extension $(L, w)$ of a valued field $(K, v)$ is immediate if it has the same value group and residue field as $(K, v)$. A valued field is (algebraically) maximal if it has no proper (algebraic) immediate extensions.

The main result of this paper is: 
Theorem 1.2. Let $(K, v)$ be a perfect, algebraically maximal valued field of characteristic $p>0$. Then the image of every additive polynomial over $K$ has the optimal approximation property.

The result follows from Theorem 3.1 which actually provides a stronger conclusion. The core observation leading to Theorem 3.1 is the fact that, in perfect fields, approximation of an arbitrary element with elements from the image of an additive polynomial in one variable can fail essentially in finitely many situations which depend only on the additive polynomial, see Corollary 2.5. It is worth noting that in positive characteristic, perfect algebraically maximal valued fields correspond exactly to tame fields, see [4]. For algebraically maximal Kaplansky Fields, the conclusion of Theorem 3.1 can be easily obtained from Theorem 6 of [7]. However, generalization of properties from Kaplansky fields to perfect fields is often an essentially different and hard problem. One obvious obstruction for such generalizations is the fact that over Kaplansky fields algebraically maximal, algebraic immediate extensions are unique and over perfect fields this is not the case.

Valued fields in positive characteristic are indeed valued fields equipped with an endomorphism, the Frobenius. This aspect has long been on the spot light but still very little is known about the contribution of the Frobenius endomorphism to the structure of a valued field of positive characteristic. I stumbled upon the issue when working with valued fields equipped with an automorphism. Valued fields equipped with an automorphism, even in residue characteristic zero, exhibit pathological behaviour very similar to that of valued fields in positive characteristic. In [2], a simple translation between the two cases is introduced, which in turn explains the similarities of these pathologies from both cases. This translation, which is essentially considering polynomials over valued fields in positive characteristic as difference polynomials, is the main technical tool leading to Corollary 2.5.

It is worth noting that the notion of extremality is related to the notion of optimal approximation but they are not quite the same thing. The original definition for extremality, in [3], turned out to ask too much and it was revised in [1]. A valued field $K$ is extremal if for every polynomial $f\left(x_{1}, \ldots, x_{n}\right) \in K\left[x_{1}, \ldots, x_{n}\right]$, the set $\left\{v f\left(a_{1}, \ldots, a_{n}\right): a_{1}, \ldots, a_{n} \in \mathcal{O}_{v}\right\}$ has a maximal element. Thus, extremality and having the optimal approximation property for the images of all polynomials (which was the original definition of extremality) are not equivalent.

This problem was brought to my attention by Franz-Viktor Kuhlmann during the Workshop on Valuation Theory in Positive Characteristic held at Nesin Mathematics Village which proved to be a wonderful environment for research and collaboration. I would like to thank Franz-Viktor Kuhlmann not only for his valuable comments on the current article but also for all the guidance and support he has provided throughout my research career.

\section{Preliminaries}

Throughout the rest of the paper $\mathcal{K}=(K, \Gamma, \boldsymbol{k}, v)$ is a valued field of characteristic $p>0$ with value group $\Gamma$ and residue field $\boldsymbol{k}$. The valuation ring is denoted by $\mathcal{O}_{v}$ and $\mathfrak{m}_{v}$ denotes the maximal ideal. The residue class map from $\mathcal{O}_{v}$ to $\boldsymbol{k}$ is $a \mapsto \bar{a}: \mathcal{O}_{v} \rightarrow \boldsymbol{k}$.

We consider the polynomial ring $K\left[\phi^{i}(x): i \in \mathbb{N}\right]$ where

$$
x=\phi^{0}(x), \phi^{1}(x), \phi^{2}(x), \ldots
$$


are distinct indeterminates. An element of this ring will be called a $\phi$-polynomial over $K$. We shall interpret $\phi$ as the Frobenius endomorphism. To be precise: for any $K$-algebra $R$ and $a \in R$, "evaluation at $a$ " is the unique $K$-algebra morphism,

$$
F \mapsto F(a): K\left[\phi^{i}(x): i \in \mathbb{N}\right] \rightarrow R
$$

sending $\phi^{i}(x)$ to $a^{p^{i}}$ for all $i \in \mathbb{N}$.

For an $(n+1)$-tuple $\boldsymbol{i}=\left(i_{0}, i_{1}, \ldots, i_{n}\right) \in \mathbb{N}^{n+1}$ we set

$$
\phi(x)^{i}:=x^{i_{0}} \cdot \phi(x)^{i_{1}} \cdots \phi^{n}(x)^{i_{n}},
$$

and $|\boldsymbol{i}|:=i_{0}+i_{1}+\cdots+i_{n}$.

A $\phi$-polynomial $F=\sum_{\boldsymbol{i}} a_{\boldsymbol{i}} \cdot \phi(x)^{\boldsymbol{i}}$ is small if each component of $\boldsymbol{i}$ is less than $p$ for all $\boldsymbol{i}$ such that $a_{\boldsymbol{i}} \neq 0$. Ordinary polynomials in one variable over $K$ can be viewed as small $\phi$-polynomials, by mapping $x^{p^{k}}$ to $\phi^{k}(x)$. See section 8 of [2], for a more detailed treatment of this translation between polynomials and small $\phi$-polynomials.

Let $x_{0}, \ldots, x_{n}, y_{0}, \ldots, y_{n}$ be distinct indeterminates, and put $\mathbf{x}=\left(x_{0}, \ldots, x_{n}\right)$, $\mathbf{y}=\left(y_{0}, \ldots, y_{n}\right)$. For a polynomial $f(\mathbf{x})$ over a field $K$ we have a unique Taylor expansion in $K[\mathbf{x}, \mathbf{y}]$ :

$$
f(\mathbf{x}+\mathbf{y})=\sum_{i} f_{(i)}(\mathbf{x}) \cdot \mathbf{y}^{i}
$$

where the sum is over all $\boldsymbol{i}=\left(i_{0}, \ldots, i_{n}\right) \in \mathbb{N}^{n+1}$, each $f_{(\boldsymbol{i})}(\mathbf{x}) \in K[\mathbf{x}]$, with $f_{(\boldsymbol{i})}=0$ for $|\boldsymbol{i}|:=i_{0}+\cdots+i_{n}>\operatorname{deg} f$, and $\mathbf{y}^{\boldsymbol{i}}:=y_{0}^{i_{0}} \cdots y_{n}^{i_{n}}$. (Also, for a tuple $a=\left(a_{0}, \ldots, a_{n}\right)$ with components $a_{i}$ in any field we put $a^{\boldsymbol{i}}:=a_{0}^{i_{0}} \cdots a_{n}^{i_{n}}$.) Thus $\boldsymbol{i} ! f_{(\boldsymbol{i})}(\mathbf{x})=\partial_{\boldsymbol{i}} f$ where $\partial_{\boldsymbol{i}}$ is the operator $\left(\partial / \partial x_{0}\right)^{i_{0}} \cdots\left(\partial / \partial x_{n}\right)^{i_{n}}$ on $K[\mathbf{x}]$, and $\boldsymbol{i} !=i_{0} ! \cdots i_{n}$ !. We construe $\mathbb{N}^{n+1}$ as a monoid under + (componentwise addition), and let $\leq$ be the (partial) product ordering on $\mathbb{N}^{n+1}$ induced by the natural order on $\mathbb{N}$. Define $\left(\begin{array}{c}\boldsymbol{i} \\ \boldsymbol{j}\end{array}\right)$ as $\left(\begin{array}{c}i_{0} \\ j_{0}\end{array}\right) \cdots\left(\begin{array}{c}i_{n} \\ j_{n}\end{array}\right) \in \mathbb{N}$, when $\boldsymbol{j} \leq \boldsymbol{i}$ in $\mathbb{N}^{n+1}$. Then:

Lemma 2.1. For $\boldsymbol{i}, \boldsymbol{j} \in \mathbb{N}^{n+1}$ we have $\left(f_{(\boldsymbol{i})}\right)_{(\boldsymbol{j})}=\left(\begin{array}{c}\boldsymbol{i}+\boldsymbol{j} \\ \boldsymbol{i}\end{array}\right) f_{(\boldsymbol{i}+\boldsymbol{j})}$.

In particular, $f_{(\boldsymbol{i})}=f$ for $|\boldsymbol{i}|=0$, and if $|\boldsymbol{i}|=1$ with $i_{k}=1$, then $f_{(\boldsymbol{i})}=\frac{\partial f}{\partial x_{k}}$. Also, $\operatorname{deg} f_{(\boldsymbol{i})}<\operatorname{deg} f$ if $|\boldsymbol{i}| \geq 1$ and $f \neq 0$.

For $f \in K\left[x_{0}, \ldots, x_{n}\right]$ as above and $F(x)=f\left(x, \ldots, \phi^{n}(x)\right)$ we have the following identity in the ring of $\phi$-polynomials in the distinct indeterminates $x$ and $y$ over $K$ :

$$
\begin{aligned}
F(x+y) & =f\left(x+y, \ldots, \phi^{n}(x+y)\right)=f\left(x+y, \ldots, \phi^{n}(x)+\phi^{n}(y)\right) \\
& =\sum_{\boldsymbol{i}} f_{(\boldsymbol{i})}\left(x, \ldots, \phi^{n}(x)\right) \cdot \boldsymbol{\phi}(y)^{\boldsymbol{i}}=\sum_{\boldsymbol{i}} F_{(\boldsymbol{i})}(x) \cdot \boldsymbol{\phi}(y)^{\boldsymbol{i}},
\end{aligned}
$$

where $F_{(i)}(x):=f_{(i)}\left(x, \ldots, \phi^{n}(x)\right)$.

In the rest of this section we let $f(x) \in K[x]$ be a nonzero polynomial. Suppose that the degree of $f$ is $i$ and $n$ is such that $i<p^{n+1}$. Let $F$ be the small $\phi$ polynomial that corresponds to $f$. Then $F \in K\left[x, \phi(x), \ldots, \phi^{n}(x)\right]$ and for any $a \in K, f(a)=F(a)$. 
Definition 2.2. ${ }^{1}$ Let $a \in K$. We that say $(f, a)$ is in $\phi$-hensel configuration if $f \notin K$ and there is $\boldsymbol{i}$ with $|\boldsymbol{i}|=1, \gamma \in \Gamma$ such that

(i) $v(F(a))=v\left(F_{(\boldsymbol{i})}(a)\right)+\boldsymbol{\phi}^{i} \gamma<v\left(F_{(\boldsymbol{j})}(a)\right)+\boldsymbol{\phi}^{\boldsymbol{j}} \gamma$ whenever $|\boldsymbol{j}|=1$,

(ii) $v\left(F_{(j)}(a)\right)+\boldsymbol{\phi}^{j} \gamma<v\left(F_{(j+l)}(a)\right)+\phi^{j+l} \gamma$ whenever $|\boldsymbol{j}|,|\boldsymbol{l}| \neq 0$ and $F_{(j)} \neq 0$.

If $(f, a)$ is in $\phi$-hensel configuration, then $F_{(\boldsymbol{j})}(a) \neq 0$ whenever $\boldsymbol{j} \neq 0$ and $F_{(\boldsymbol{j})} \neq 0$. Hence $F(a)=f(a) \neq 0$ and $\gamma$ as above satisfies

$$
v(F(a))=\min _{|\boldsymbol{j}|=1} v\left(F_{(\boldsymbol{j})}(a)\right)+\boldsymbol{\phi}^{\boldsymbol{j}} \gamma,
$$

so is unique and we set $\gamma(f, a):=\gamma$.

Lemma 2.3. Suppose that $\Gamma$ is p-divisible. Let $f(x)=a_{0} x+a_{1} x^{p}+\cdots+a_{n} x^{p^{n}}$ be a nonzero additive polynomial over $K$. There are $\theta_{1}, \ldots, \theta_{k} \in \Gamma$ such that for every nonzero $c \in K$ if $v(c) \neq \theta_{1}, \ldots, \theta_{k} \in \Gamma$, then $(f(x)+c, 0)$ is in $\phi$-hensel configuration.

Proof. As $f$ is nonzero there is at least one $i \in\{0, \ldots, n\}$ with $a_{i} \neq 0$ and for such $i$ define

$$
\Lambda_{i}: \Gamma \rightarrow \Gamma, \quad \gamma \mapsto v\left(a_{i}\right)+p^{i} \gamma .
$$

Note that $\Lambda_{i}$ is bijective as $\Gamma$ is $p$-divisible. Since each $\Lambda_{i}$ is increasing at a rate of $p^{i}$, the graphs of $\Lambda_{i}$ have finitely many intersection points. Therefore for all $\theta \in \Gamma$ there is a unique $\gamma \in \Gamma$ such that

$$
\theta=\min _{i} \Lambda_{i}(\gamma)
$$

and except for finitely many values of $\gamma \in \Gamma$ we have $\Lambda_{i}(\gamma) \neq \Lambda_{j}(\gamma)$ for $i \neq j$. So there are $\theta_{1}, \ldots, \theta_{k} \in \Gamma$ such that the minimum above is achieved at a single index $i$.

Now assume that $c$ is a nonzero element of $K$ with $v(c) \neq \theta_{1}, \ldots, \theta_{k}$. Then there is $i \in\{0, \ldots, n\}$ and $\gamma \in \Gamma$ such that

$$
v(c)=\Lambda_{i}(\gamma)<\Lambda_{j}(\gamma)
$$

whenever $a_{j} \neq 0$ and $i \neq j$. Moreover if $F(x)$ is the $\phi$-polynomial which corresponds to $f(x)+c$ then $F_{(\boldsymbol{j})}=0$ whenever $|\boldsymbol{j}|>1$. So $(f(x)+c, 0)$ is in $\phi$-hensel configuration.

Lemma 2.4. Suppose that $\Gamma$ is divisible and $\boldsymbol{k}$ is perfect. If $f(x) \in K[x]$ and $a \in K$ with $(f, a)$ in $\phi$-hensel configuration then there is $b \in K$ such that $v(b-a)=\gamma(f, a)$ and $v(f(b))>v(f(a))$.

Proof. Let $F$ be the small $\phi$-polynomial that corresponds to $f$ and $\gamma=\gamma(f, a)$. Pick $\epsilon \in K$ with $v(\epsilon)=\gamma$. Let $b=a+\epsilon u$ where $u \in K$ is to be determined later, we only impose $v(u)=0$ for now. Consider

$$
F(b)=F(a)+\sum_{|i| \geq 1} F_{(i)}(a) \cdot \phi(b-a)^{i} .
$$

\footnotetext{
${ }^{1}$ This definition is not the same as the definition of $\phi$-hensel configuration that appears in [2] which was devised to deal with Kaplansky fields.
} 
Therefore $F(b)=F(a) \cdot\left(1+\sum_{|\boldsymbol{i}| \geq 1} c_{\boldsymbol{i}} \cdot \phi(u)^{\boldsymbol{i}}\right)$, where

$$
c_{i}=\frac{F_{(i)}(a) \cdot \phi(\epsilon)^{i}}{F(a)} .
$$

Since $v(\epsilon)=\gamma$, we see that there is $\boldsymbol{i}$ with $|\boldsymbol{i}|=1$ such that $v\left(c_{\boldsymbol{i}}\right)=0$, and $v\left(c_{\boldsymbol{j}}\right)>0$ for all $\boldsymbol{j} \neq \boldsymbol{i}$. Since $\boldsymbol{k}$ is perfect we can take $u$ such that $\bar{u}$ is a solution of

$$
1+\overline{c_{i}} \cdot \bar{\phi}(x)^{i}=0 .
$$

Then $v(u)=0$, and $v(b-a)=\gamma(f, a)$ and $v(f(b))>v(f(a))$.

Corollary 2.5. Suppose that $\mathcal{K}$ is a perfect algebraically maximal valued field. Let $f(x)=a_{0} x+a_{1} x^{p}+\cdots+a_{n} x^{p^{n}}$ be a nonzero additive polynomial over $K$. Then there are $\theta_{1}, \ldots, \theta_{k} \in \Gamma$ such that for every $c \in K$ either

- $\max \{v(f(a)-c): a \in K\}=\theta_{i}$ for some $i=1, \ldots, k$; or

- the equation $f(x)-c=0$ has a solution in $K$.

Proof. Note that $\Gamma$ is $p$-divisible and $\boldsymbol{k}$ is perfect as $K$ is perfect. Let $\theta_{1}, \ldots, \theta_{k}$ be as in the conclusion of Lemma 2.3 for the additive polynomial $f(x)$. Let $c \in K$. Since $\mathcal{K}$ is algebraically maximal, $\{v(f(a)-c): a \in K\} \subset \Gamma \cup\{\infty\}$ has a maximal element. Assume that $f(x)-c=0$ has no solution in $K$ and let $a^{\prime} \in K$ be such that $v\left(f\left(a^{\prime}\right)-c\right)=\max \{v(f(a)-c): a \in K\} \in \Gamma$. Suppose that $v\left(f\left(a^{\prime}\right)-c\right) \neq \theta_{1}, \ldots, \theta_{k}$. Then by Lemma 2.3, $\left(f(x)+f\left(a^{\prime}\right)-c, 0\right)$ is in $\phi$-hensel configuration. Therefore Lemma 2.4 gives $b$ with

$$
v\left(f(b)+f\left(a^{\prime}\right)-c\right)=v\left(f\left(b+a^{\prime}\right)-c\right)>v\left(f\left(a^{\prime}\right)-c\right),
$$

contradiction.

\section{Main Result}

Theorem 3.1. Suppose that $\mathcal{K}$ is a perfect algebraically maximal valued field. Let $h\left(x, y_{1}, \ldots, y_{m}\right) \in K\left[x, y_{1}, \ldots, y_{m}\right]$ be such that

$$
h\left(x, y_{1}, \ldots, y_{m}\right)=f(x)+g\left(y_{1}, \ldots, y_{m}\right),
$$

where $f(x) \in K[x]$ is a nonzero additive polynomial, and $g\left(y_{1}, \ldots, y_{m}\right) \in K\left[y_{1}, \ldots, y_{m}\right]$. Then the image of $h$ has the optimal approximation property.

Proof. Let $c \in K$ and assume that for all $\left(a, b_{1}, \ldots, b_{m}\right) \in K^{m+1}$ if

$$
h\left(a, b_{1}, \ldots, b_{m}\right)-c \neq 0
$$

then there is $\left(a^{\prime}, b_{1}^{\prime}, \ldots, b_{m}^{\prime}\right) \in K^{m+1}$ such that

$$
v\left(h\left(a^{\prime}, b_{1}^{\prime}, \ldots, b_{m}^{\prime}\right)-c\right)>v\left(h\left(a, b_{1}, \ldots, b_{m}\right)-c\right) .
$$

The result will follow by finding a solution to the equation $h\left(x, y_{1}, \ldots, y_{m}\right)-c=0$.

Let $\theta_{1}, \ldots, \theta_{k}$ be as in the conclusion of Corollary 2.5 for the additive polynomial $f(x)$. Let $\left(a, b_{1}, \ldots, b_{m}\right) \in K^{m+1}$ and assume $h\left(a, b_{1}, \ldots, b_{m}\right)-c \neq 0$. We have

$$
h\left(a+x, b_{1}, \ldots, b_{m}\right)-c=f(x)+h\left(a, b_{1}, \ldots, b_{m}\right)-c .
$$

By Corollary 2.5, either we have a solution to the equation

$$
h\left(a+x, b_{1}, \ldots, b_{m}\right)-c=f(x)+h\left(a, b_{1}, \ldots, b_{m}\right)-c=0
$$


or $\max \left\{v\left(f(a+d)+h\left(a, b_{1}, \ldots, b_{m}\right)-c\right): d \in K\right\}=\theta_{i}$ for some $i \in\{1, \ldots, k\}$. By our assumption above the latter is not possible, so we obtain the result.

It is now straightforward to obtain Theorem 1.2 .

\section{REFERENCES}

[1] S. Azgin, F-V. Kuhlmann, and F. Pop. Characterization of extremal valued fields. Proc. Amer. Math. Soc., 43(140):1535-1547, 2012.

[2] Salih Azgin. Valued fields with contractive automorphism and Kaplansky fields. J. Algebra, 324(10):2757-2785, 2010.

[3] Yu. L. Ershov. Extremal valued fields. Algebra Logika, 43(1):582-588, 2004.

[4] F.-V. Kuhlmann. The algebra and model theory of tame valued fields. Preprint.

[5] F.-V. Kuhlmann. Elementary properties of power series fields over finite fields. J. Symb. Logic, 43(66):771-791, 2001

[6] F.-V. Kuhlmann. A classification of artin schreier defect extensions and a characterization of defectless fields. Illinois J. Math., 43(54):397-448, 2010.

[7] Franz-Viktor Kuhlmann. Additive polynomials and their role in the model theory of valued fields. In Proceedings of the Workshop and Conference on Logic, Algebra, and Arithmetic, held October 18-22, 2003, Lecture Notes in Logic 26, pages 160-203. 2006.

[8] L. van den Dries and F.-V. Kuhlmann. Images of additive polynomials in $\mathbb{F}_{q}((t))$ have the optimal approximation property. Can. Math. Bulletin, 45:71-79, 2002.

Mathematics Research and Teaching Group, Middle East Technical University, Northern Cyprus Campus, Kalkanli, Güzelyurt, KKTC, Mersin 10, Turkey 